Article

\title{
A Mutual Capacitance Touch Readout IC with Synchronization in Touch and Mobile Display Driving for High Refresh Rate AMOLED Panels
}

\author{
Seunghoon Ko
}

Citation: Ko, S. A Mutual Capacitance Touch Readout IC with Synchronization in Touch and Mobile Display Driving for High Refresh Rate AMOLED Panels. Micromachines 2021, 12, 922. https://doi.org/ $10.3390 / \mathrm{mi} 12080922$

Academic Editor:

Takafumi Fukushima

Received: 30 June 2021

Accepted: 29 July 2021

Published: 31 July 2021

Publisher's Note: MDPI stays neutral with regard to jurisdictional claims in published maps and institutional affiliations.

Copyright: (C) 2021 by the author Licensee MDPI, Basel, Switzerland. This article is an open access article distributed under the terms and conditions of the Creative Commons Attribution (CC BY) license (https:/ / creativecommons.org/licenses/by/ $4.0 /)$.
Department of Electronic Materials Engineering, Kwangwoon University, Seoul 01897, Korea; shko@kw.ac.kr

\begin{abstract}
This paper presents a mutual capacitance touch readout IC architecture for $120 \mathrm{~Hz}$ highrefresh-rate AMOLED displays. In high-refresh-rate AMOLED panels, whole pixels in a horizontal line should be updated without any time-sharing with each other, leading to an amplified display noise on touch screen panel (TSP) electrodes. The proposed system architecture mitigates severe display noise by synchronizing the driving for the TSP and AMOLED pixel circuits. The proposed differential sensing technique, which is based on noise suppression in reference to mutual capacitance channels, minimizes common-mode display noise. In the front-end circuit, intrinsic circuit offset is cancelled by a chopping scheme, which correlates to the phase of the driving signals in the TSP driver and operating clocks of the front-end. Operating at a $120 \mathrm{~Hz}$ scan-rate, it reduces display noise by more than $11.6 \mathrm{~dB}$ when compared with the conventional single-ended TSP sensing method. With a built-in 130-nm CMOS, a prototype IC occupies an area of $8.02 \mathrm{~mm}^{2}$ while consuming $6.4-\mathrm{mW}$ power from a $3.3 \mathrm{~V}$ analog voltage supply.
\end{abstract}

Keywords: touch screen panel; differential readout system; capacitance sensor

\section{Introduction}

Nowadays, with increasing demand for high-quality mobile device displays, display vendors strive to advance their technology featuring ultra-thin, transparent, and fast response time displays. Recent significant progress in mobile display driver integrated chips (MDDI) and pixel circuit technology results in the "seamless display", or an introduction of a high refresh rate [1] in mobile devices where the MDDI updates each display frame within 8.33 milliseconds. In addition, a variable refresh rate (VRR) [2] is employed in high-end smartphones, in which the refresh rate is adjusted automatically depending on the display content. A refresh rate less than $10 \mathrm{~Hz}$ can be maintained for a static display image such as an always-on-display (AOD) function [3], while a fast frame transition of $120 \mathrm{~Hz}$ is needed for high-resolution gaming content.

Figure 1a illustrates an equivalent circuit and the stack-up of an active-matrix organic light-emitting diode (AMOLED) pixel and touch screen panel (TSP) when MDDI operates. In an on-cell display panel [4], TSP electrodes are deposited and attached onto a display pixel layer using optically clear adhesive (OCA) in a separate manufacturing process. However, in case of AMOLED panels, TSP electrodes are directly printed over the encapsulation glass which protects AMOLED pixel from oxidation and physical damage. Therefore, the ground plane for AMOLED pixel should be shared with TSP readout system. Active shielding in the ground plane $[4,5]$ cannot be applied to AMOLED display systems, which is more vulnerable to display noise and the effects of TSP parasitic capacitance.

The MDDI, consisting of a binary amplifier (B-Amp) and a resistive digital-to-analog converter (R-DAC), provides RGB signals, and the thin-film transistor circuit in the AMOLED receives and updates these signals into the array of pixels (P[H:1]). Demultiplexers (Demux) at the MDDI output have been widely adopted for low-resolution and low-refresh-rate displays. They ease the power and area budget of commercialized MDDIs. 
With a 3-to-1 Demux, the MDDI can refresh three times more pixels than each sub-block in the MDDI (MDDI[H/3:1]) during a horizontal blanking period $\left(\mathrm{T}_{\mathrm{HSYNC}}\right)$. Figure $1 \mathrm{~b}$ illustrates the timing diagram of an MDDI when a zebra pattern repeating white and black lines is updated with a 3-to-1 Demux. During $\mathrm{T}_{\mathrm{HSYNC}}$, the pixel update timing can be divided into 3 phases. For example, the demultiplexer outs of D_Out[H/3:1] are refreshed into the $\mathrm{P}[\mathrm{H} / 3: 1]$ during one third of the $\mathrm{T}_{\mathrm{HSYNC}}$. In contrast, for a $120 \mathrm{~Hz}$ refresh rate display such as full high-definition (FHD) or wide quad high-definition (WQHD) high-end displays having $1920 \times 1080$ and $2560 \times 1440$ resolution, there is no room for time-interleaving the AMOLED pixel driving during a $\mathrm{T}_{\text {HSYNC }}$. Column-parallel B-Amps and R-DACs per each AMOLED pixel should be integrated into the MDDI to update whole pixels ( $\mathrm{P}[\mathrm{H}: 1])$ in a horizontal line at the same time during a $\mathrm{T}_{\mathrm{HSYNC}}$. Although this technique can enhance the refresh rate of a display update, substantial display interferences are also generated due to the simultaneous transition of outputs of MDDI[H:1]. These interferences are directly added to the TSP electrodes through the series of $\mathrm{C}_{\mathrm{P}_{-} \mathrm{MDDI}}$ and $\mathrm{C}_{\mathrm{P}_{-} \mathrm{TSP}}$, degrading the signal-to-noise ratio (SNR) when we measure the mutual capacitance $\left(\mathrm{C}_{\mathrm{M}}\right)$ between the transmitter (TX) and receiver (RX) TSP electrodes.

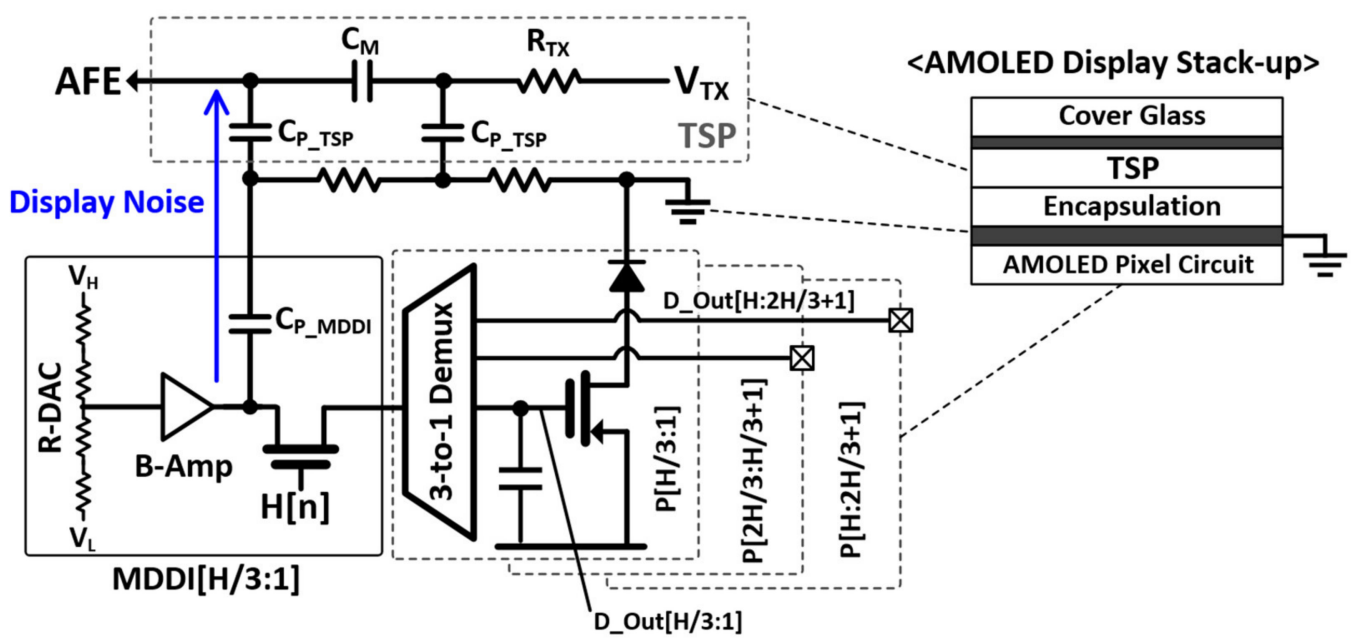

(a)

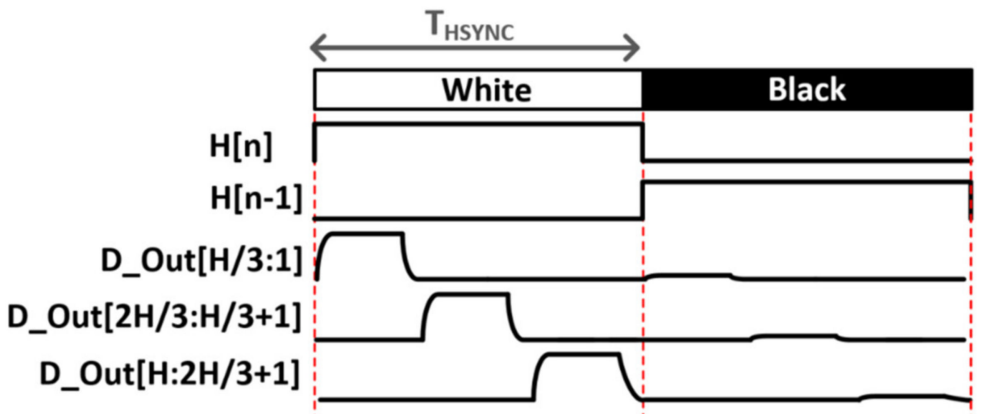

(b)

Figure 1. (a) An equivalent circuit consisting of an AMOLED, a TSP and an MDDI, and (b) a timing diagram of the MDDI operation using a 3-to-1 demultiplexer.

Therefore, high-resolution, high-refresh-rate displays lead to an increased burden for the analog front-end (AFE) of a TSP readout system and its post processing algorithm. Several researchers $[6,7]$ have proposed using a frequency hopping scheme to avoid noisy frequency bands when choosing the TSP driving frequency. However, they would suffer from an in-band noise disturbance, the amplitude and frequency of which vary according to the display contents in a high-refresh-rate display. The works in [8-11] use differential 
front-end architecture to cancel out the common-mode interference, but the importance and difficulty of choosing a reference for capacitance reconstruction have not been addressed.

This article presents a column-parallel differential sensing TSP readout system with a noise-free capacitance reconstruction technique. It enables substantial noise reduction over the reference capacitance with synchronization of the display and TSP driving. The readout IC also introduces the use of different orthogonal sequences for differential and reference TSP capacitance sensing. The proposed readout system senses a total of $31 \times 16$ TSP mutual capacitances and operates at a $120 \mathrm{~Hz}$ TSP scan rate using a $3.3 \mathrm{~V}$ power supply. This article is organized as follows. Section 2 presents the operation principle of the proposed differential sensing architecture with the noise-free reference capacitance sensing technique. Section 3 presents the circuit implementation. The measurements, results, and discussion are provided in Section 4. Finally, Section 5 concludes this article.

\section{Proposed Differential Sensing Scheme}

\subsection{Display Noise Measurement}

Figure 2 shows the display noise measured on a TSP electrode when a zebra pattern is updated onto a $2400 \times 1080 \mathrm{FHD}$ plus panel with a $120 \mathrm{~Hz}$ refresh rate. One horizontal (1-H) zebra pattern which alternates horizontal black and white colors at a time interval of $\mathrm{T}_{\text {HSYNC }}\left(=1 / \mathrm{f}_{\text {HSYNC }}\right)$ was used for this measurement. In this case, we can approximately calculate the display noise frequency from the product of the refresh rate $(120 \mathrm{~Hz})$ and horizontal resolution (2400) of the panel. The maximum signal swing of the output of the MDDI (MDDI_out[1:H]) occurs when it goes from black to white or from white to black horizontal lines. At those moments, display noise is also maximized. The noise waveform is a form of the derivative of a rectangular MDDI_Out, since the noise current flows across the series of $C_{P_{-} \text {MDDI }}$ and $C_{P_{-} \text {TSP }}$ (Figure $1 \mathrm{a}$ ). The harmonics of $f_{\text {HSYNC }}$ also exist in the noise signal frequency. Note that the noise frequency highly depends on the display contents to be updated, so it cannot be expected or removed by the frequency hopping scheme proposed in prior works. Compared with the HD panel which executes time-interleaved updates using 3-to-1 Demux in a $\mathrm{T}_{\mathrm{HSYNC}}$, the noise voltage increased by three times $(9 \mathrm{~dB})$, incurring a large noise penalty with a high-resolution, high-refresh-rate display.
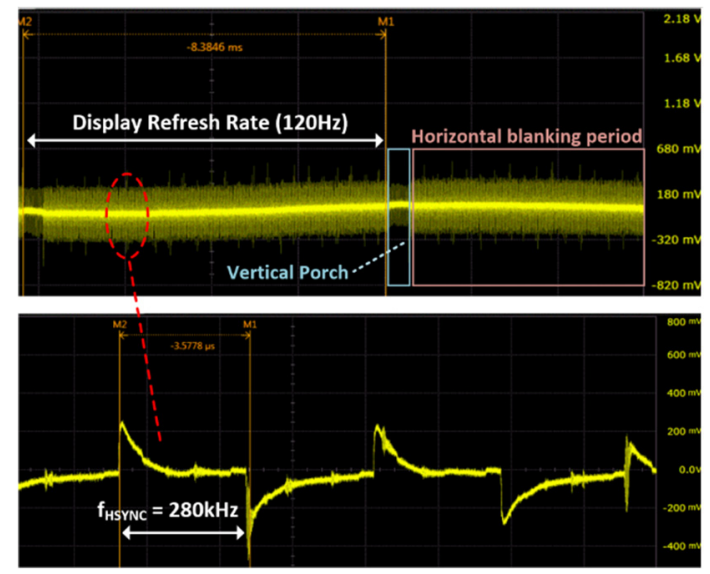

(a)

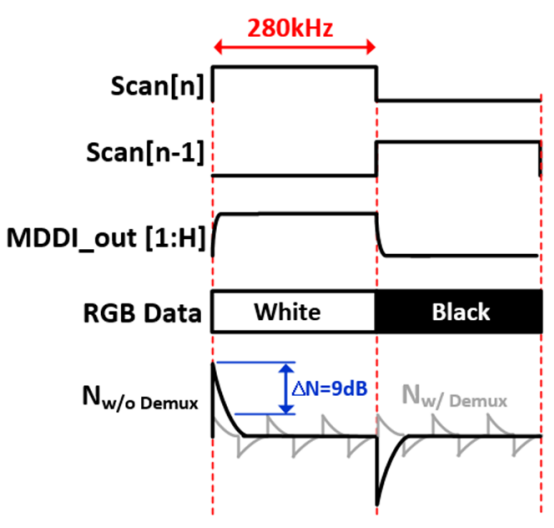

(b)

Figure 2. (a) Measured display noise with an FHD+ AMOLED display, and (b) comparison of display noise levels with a zebra image without Demux and with a 3-to-1 Demux configuration.

\subsection{Basic Principle of Differential Sensing}

The detailed operation of differential TSP sensing is shown in Figure 3. The TSP consists of M TX electrodes and N RX electrodes. An entire TSP channel is divided into the reference capacitance channels $\left(\mathrm{C}_{\mathrm{M}(1,1)}-\mathrm{C}_{\mathrm{M}(\mathrm{M}, 1)}\right)$ in a, $\mathrm{RX}$ electrode, and the remaining capacitance channels $\left(\mathrm{C}_{\mathrm{M}(1,2)}-\mathrm{C}_{\mathrm{M}(\mathrm{M}, \mathrm{N})}\right)$ for differential sensing. The capacitance channel or mutual capacitance in a TSP is generated at the cross-section of each of the TX and 
RX electrodes. During the sensing phases for both differential and reference capacitance, the driver stimulates the $\mathrm{TX}$ electrodes (from $\mathrm{TX}_{1}$ to $\mathrm{TX}_{\mathrm{M}}$ ) sequentially or concurrently using encoded $T X$ signals (from $\mathrm{V}_{\mathrm{TX}, 1}$ to $\mathrm{V}_{\mathrm{TX}, \mathrm{M}}$ ). These signals are coupled through the mutual capacitance channel and measured at the RX electrode by the AFE. Therefore, touch sensitivity becomes proportional to the product of the mutual capacitance and the signal swing of the driver output. A simple dynamic buffer-generating square wave can be used as the TSP driver, reducing the design complexity. At the AFE, column-parallel differential sensing is usually employed to suppress common-mode noises, which include any external noises as well as the display interference. A fully or pseudo-differential trans-impedance amplifier or switched-capacitor integrator are used as a first AFE stage to detect only the capacitance difference $\left(\Delta \mathrm{C}_{\mathrm{M}}\right)$. At that moment, besides the $\Delta \mathrm{C}_{\mathrm{M}}$, the interferences are frozen on the first stage but are cancelled differentially. Post-processing of capacitance data to extract an exact touch coordinate requires that the $\Delta C_{M}$ be recovered to a single-ended $\mathrm{C}_{\mathrm{M}}$. We can execute an integral of the $\Delta \mathrm{C}_{\mathrm{M}}$ of a column in a horizonal direction, which is based on the reference capacitance channels $C_{M(1,1)}-C_{M(M, 1)}$, in an RX electrode. Thus, the reference RX electrode acts like an integral basis and the recovered single-ended signals $\left(S_{R C V}(m, n)\right)$ for the $\mathrm{C}_{\mathrm{M}(\mathrm{m}, \mathrm{n})}$ can be expressed as

$$
S_{R C V}(m, n)=S_{m, 1}+\gamma N_{m, 1}+\sum_{k=2}^{n}\left(\Delta S_{m, k}+\Delta N_{m, k}\right)
$$

where we assume that $S_{m, 1}$ and $N_{m, 1}$ are the capacitance and noise signals at the crosssection of the $m$ th TX, and the 1st RX electrodes measured in a single-ended manner and $\Delta S_{m, k}$ and $\Delta N_{m, k}$ are the difference values of $S_{m, k}-S_{m, k-1}$ and $N_{m, k}-N_{m, k-1}$, respectively. The attenuation ratio $(\gamma)$ of $N_{m, 1}$ needs special attention. If $\gamma$ is equal to zero, $\Delta N_{m, k}$ can be suppressed by the differential operation. Otherwise, $N_{m, 1}$ can be considered as a global noise component across all TSP channels in differential sensing.

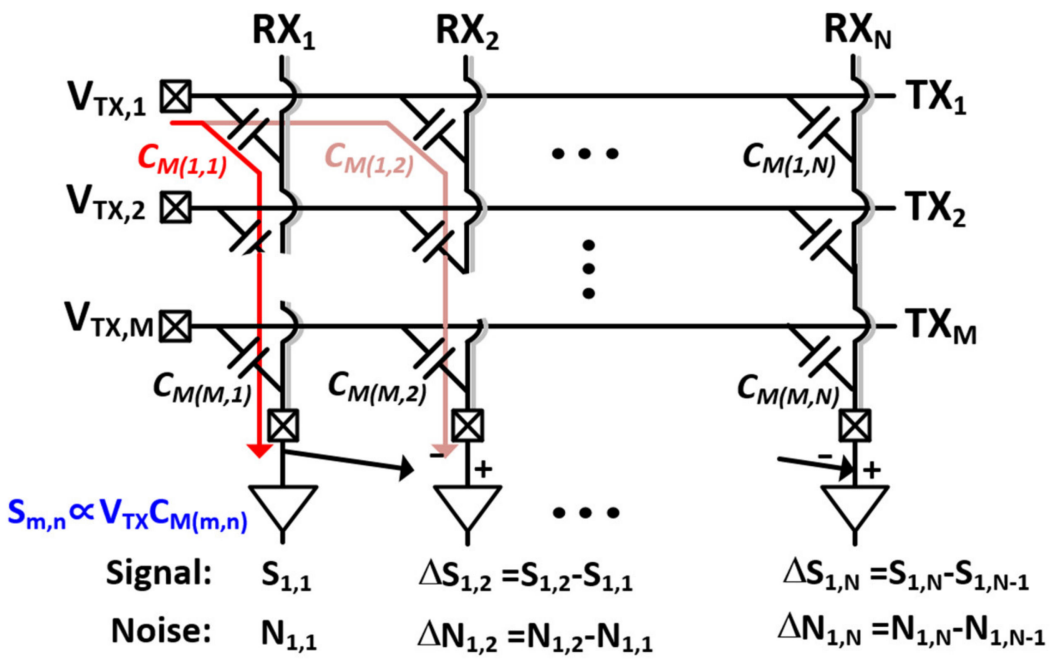

Figure 3. Illustration of the operation principle of a differential TSP sensing scheme.

This equation inspires us to choose and set the noise-immune reference capacitance channels, noise to which directly adds to the reconstructed capacitance value. Thus, the degree of noise suppression in a differential TSP sensing readout system depends on how much of the noise voltage is frozen in the reference capacitance channels.

\subsection{Detailed Operation of the Proposed Scheme}

Figure 4 provides a timing diagram to illustrate our proposed differential TSP sensing mechanism. The display frame update repeats itself every vertical synchronization signal (VSYNC), where an entire MDDI operation cycle is divided into two phases: the vertical 
front porch $\left(\mathrm{T}_{\mathrm{VP}}\right)$ from $t_{1}$ to $t_{2}$, and the horizontal blanking period $\left(\mathrm{T}_{\mathrm{HP}}\right)$ from $t_{2}$ to $t_{3}$. During $\mathrm{T}_{\mathrm{VP}}$, the voltage of the cathode plane in the AMOLED is changed, which, in a mobile device, is dependent on the brightness of the display. Since the rate of change of the voltage is too slow, the noise current from the cathode plane to the TSP electrode, which passes through the parasitic capacitance of TSP $\left(\mathrm{C}_{\mathrm{P}_{\mathrm{Z}} \mathrm{TSP}}\right)$ (Figure 1a), is also insignificant. The AMOLED pixel voltage begins to vary from $t_{2}$. As with high-refresh-rate, highresolution panels, all RGB pixels $\mathrm{P}[\mathrm{H}: 1]$ in a horizontal line should be updated in a $\mathrm{T}_{\mathrm{HSYNC}}$, which is synchronous to the horizontal synchronization signal (HSYNC). In this case, the rising and falling time of the MDDI_out[H:1] should also decrease, leading to an amplified noise current insertion through the series of $\mathrm{C}_{\mathrm{P}_{-} \mathrm{MDDI}}$ and $\mathrm{C}_{\mathrm{P}_{-} \mathrm{TSP}}$ to the TSP electrode. Total display noise is also proportional to the number of pixels $(\mathrm{H})$ in a horizontal line to be updated. In this design, we take careful consideration in dealing with the timing of the reference and differential capacitance driving. The reference capacitance channels in an $\mathrm{RX}$ electrode are driven by $\mathrm{TX}_{\mathrm{REF}}<30: 0>$ during $\mathrm{T}_{\mathrm{VP}}$. This is because the display noise is negligible compared with that during $\mathrm{T}_{\mathrm{HP}}$. On the other hand, a column-parallel capacitance difference is measured during $\mathrm{T}_{\mathrm{HP}}$. The common noise voltage freezes to both the positive and negative paths of the differential sensing AFE, but is cancelled differentially. By separating the sensing phases of the reference and differential capacitance, an improved SNR is achieved for its reference capacitance against the display noise, and sufficiently long $\mathrm{T}_{\mathrm{HP}}$ helps to suppress any external noises $[12,13]$ besides the display noise.

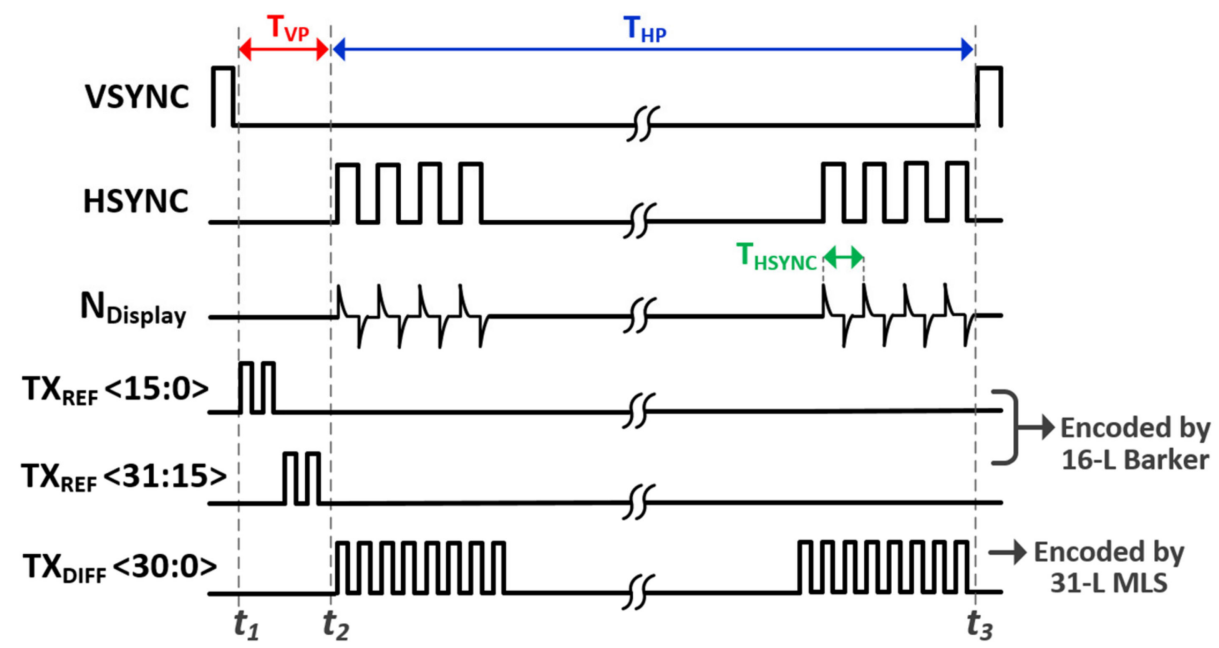

Figure 4. Timing diagram for illustrating the proposed differential TSP sensing scheme.

Different orthogonal sequences, $\mathrm{TX}_{\mathrm{REF}}<30: 0>$ and $\mathrm{TX}$ DIFF $<30: 0>$, were employed for the driving of the reference and differential capacitances. When we introduced the orthogonal driving into the TSP readout system [14-16], we faced a tradeoff between dynamic range and crosstalk. Although the multi-channel driven concept increased the sensing time for each TSP channel, leading to increased sensitivity, total capacitance to be processed by the AFE also increased. This limited the dynamic range of the AFE and increased the burden to the operation amplifier from the trans-impedance amplifier or the switched capacitor integrator, which are the first stage of AFE. For a Hadamard orthogonal sequence, the vertical code summation becomes equal to the number of orthogonal sequences. One method is to use another orthogonal sequence that minimizes the code summation, but it is at the expense of increased residue between the TSP channels in adjacent TX electrodes, where the residue can be defined by the inner product of orthogonal sequences. In case of the n-length (n-L) maximum-length orthogonal sequence (MLS) $[17,18]$, the inner product is equal to $\mathrm{n}$ when inner product with itself is done. However, the inner product for different MLSs is reduced to 1, regardless of length. Thus, the crosstalk can be calculated to 1 divided by $n$, or by the code-length of the MLS. 
In a practical design, when an FHD and an AMOLED having a 31 TX and 16 RX electrode TSP was evaluated, we applied a different orthogonal encoding method during the $\mathrm{T}_{\mathrm{VP}}$ and $\mathrm{T}_{\mathrm{HP}}$, as shown in Figure 5. Firstly, during $\mathrm{T}_{\mathrm{VP}}$ for reference capacitance sensing, there are also two sensing phases: the upper TSP sensing phase, and the lower TSP sensing phase. The reasons are twofold. First, the panel bandwidth ( $\mathrm{f}_{\mathrm{TBW}} \mathrm{UPPER}$ ) of the upper TSP area is much smaller than that of the lower TSP area. Thus, the TX driver experiences the greatest delay when it transfers the charge of $C_{M(1,1)}$ (in Figure 3) from TX [1] to RX [1]. This phase mismatch would disrupt the orthogonality if we were to choose a higher TX frequency free from external noises besides the display noise. Second, code summation should be minimized while maintaining perfect orthogonality between each TX electrode, which is highly desirable to set the integral basis for differential sensing. To achieve a reasonable tradeoff, a 16-length baker sequence $[19,20]$ was applied to $T X_{R E F}<0: 15>$ and $\mathrm{TX}_{\mathrm{REF}}<15: 31>$. Here, $\mathrm{TX}_{\mathrm{REF}}<15>$ was retransmitted for the lower TSP area, which can balance the code summation to four without incurring crosstalk.

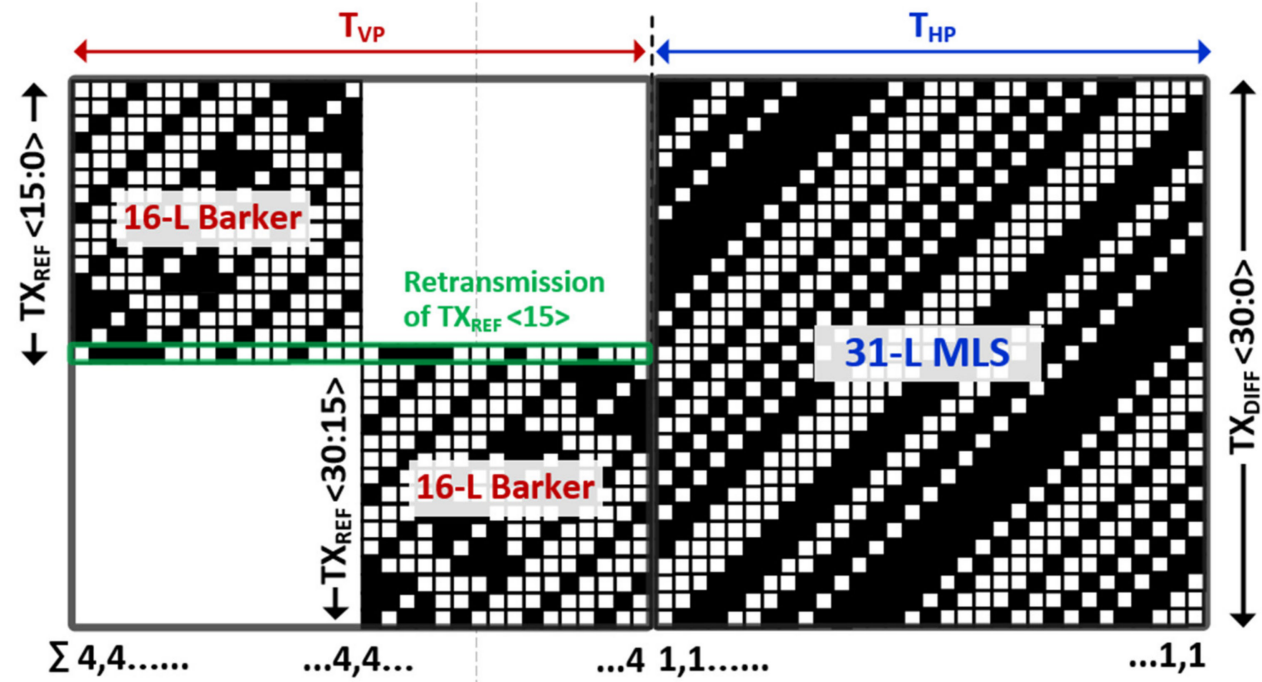

Figure 5. Application of different orthogonal sequences in reference and differential capacitance sensing.

During $\mathrm{T}_{\mathrm{HP}}$, all TX electrodes are driven simultaneously by the 31-L MLSs, which is desirable for two reasons. First, display noise due to the RGB pixel updates in the $\mathrm{T}_{\mathrm{HP}}$ does not saturate the AFE since the code summation is reduced to one mutual capacitance unit $\left(\mathrm{C}_{\mathrm{M}}\right)$. Second, the SNR increases by the square root of 31 compared with that of sequential driving. Although the TX frequency is restricted to be much lower than the $\mathrm{f}_{\mathrm{TBW}}$ _UPPER and crosstalk exists between the TX electrodes, this noise penalty can be suppressed by permitting differential capacitance sensing.

In the TSP readout system, the inner product for $\mathrm{TX}_{\mathrm{REF}}<30: 0>$ and $\mathrm{TX}_{\mathrm{DIFF}}<30: 0>$ can be found to be

$$
\begin{gathered}
\mathrm{TX}_{\mathrm{REF}}<i>\cdot \mathrm{TX}_{\mathrm{REF}}<j>=\left\{\begin{array}{c}
16(i=j) \\
0(i \neq j)
\end{array}\right. \\
\mathrm{TX}_{\mathrm{DIFF}}<i>\cdot \mathrm{TX}_{\mathrm{DIFF}}<j>=\left\{\begin{array}{c}
31(i=j) \\
1(i \neq j)
\end{array}\right.
\end{gathered}
$$

\section{Circuit Implementations}

\subsection{Whole Readout System Architecture}

Figure 6 shows the proposed TSP sensing architecture, where it senses the mutual capacitance of 31 TX electrodes and 16 RX-TSP electrodes. It is comprised of a TSP driver, a switched capacitor integrator for capacitance-to-voltage (C2V) conversion, a successive approximation register (SAR) analog-to-digital converter (ADC), and decoding and reconstruction logic. During the reference capacitance sensing phase $\left(\mathrm{T}_{\mathrm{VP}}\right)$, the TSP driver 
stimulates the TX electrode by $\mathrm{TX}_{\mathrm{REF}}<30: 0>$. The TX carrier frequency is encoded by a 16- $\mathrm{L}$ Barker sequence, and the upper and lower TSP areas are driven separately. When the $\mathrm{T}_{\mathrm{VP}}$ finishes, all TX electrodes are driven simultaneously by $\mathrm{TX}_{\mathrm{DIFF}}\langle 30: 0\rangle$, which is encoded by 31-L MLS. To suppress external and display coupling noise, the sensing time should be sufficiently long, thus occupying most of the $\mathrm{T}_{\mathrm{HP}}$ for differential sensing. At the same time, the front-end executes column-parallel TSP sensing with $16 \mathrm{C} 2 \mathrm{~V}$ converters (C2V[15:0]). Besides conversion, a $\mathrm{C} 2 \mathrm{~V}$ converter acts as a discrete-time bandpass filter whose passband is centered on the TX frequency. The base capacitance, which is unchanged by the touch of a finger, is cancelled through a current compensation technique, where the sink ( $\left.\mathrm{I}_{\mathrm{SK}}\right)$ or source ( $\mathrm{ISC}_{\mathrm{SC}}$ ) current is provided to the AC-modulated capacitance signals at the $\mathrm{C} 2 \mathrm{~V}$ input. The voltage of the virtual ground of the $\mathrm{C} 2 \mathrm{~V}$ converter is fixed to half of the supply voltage, but exhibits an offset. In case of a panel having a large $C_{P_{-} T S P}$, it can saturate the output of a C2V. This risk can be removed by a chopping scheme where the phase of a TX signal is synchronous to the closing of $\phi_{1}, \phi_{2}$, and a 16-to-1 bidirectional ADC multiplexer (Mux).

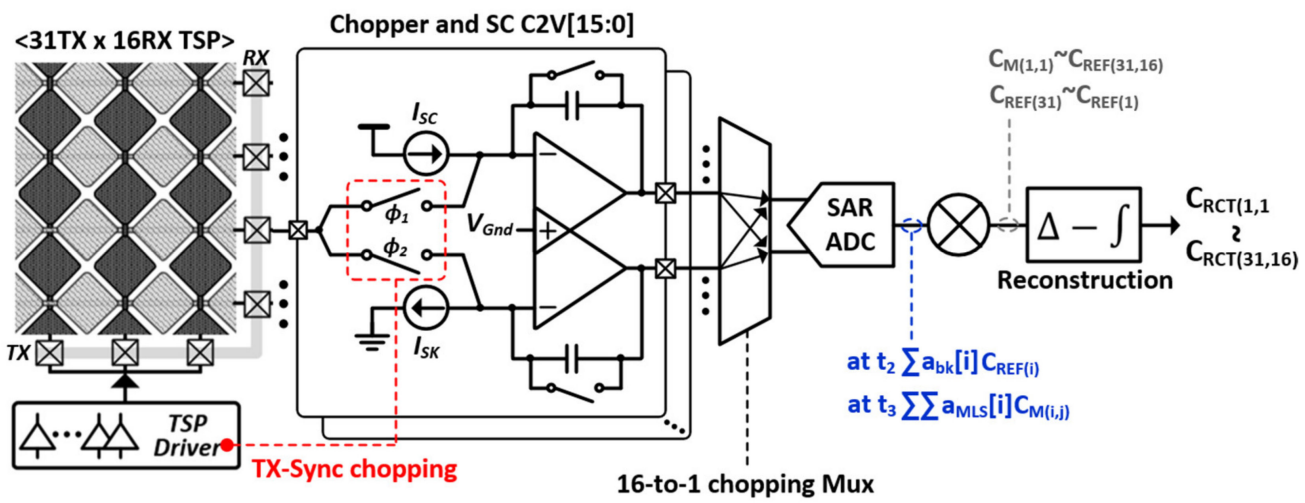

Figure 6. Proposed TSP-DDI synchronous differential front-end architecture.

The system adopts a differential sensing scheme processed in a digital domain. The reasons are twofold. First, in a practical design, we should sense both a single ended reference capacitance and the capacitance difference. Thus, we can choose the same input stage to sense both. Moreover, compared with previous work enabling higher-order band pass filtering with multiple AFE stages in an analog domain, it can ease the power and area budget of the IC with only two op-amps in the CDS. Second, compared with previous works, it can increase the sensing time by two times compared with differential AFE sensing. For simplicity of explanation, let us consider when the differences of $C_{M(1,1)}$, $\mathrm{C}_{\mathrm{M}(1,2)}$, and $\mathrm{C}_{\mathrm{M}(1,3)}$ are sensed directly at the AFE. In this case, after the difference between $C_{M(1,1)}$ and $C_{M(1,2)}$ is sensed, that of $C_{M(1,2)}$ and $C_{M(1,3)}$ should be sensed sequentially to reconstruct the single-ended capacitance. This lowers the sensitivity by $3 \mathrm{~dB}$ compared with differential processing in the digital domain. This way, the insufficient noise filtering of differential sensing in digital domain can be supplemented. With a reference capacitance of $C_{R E F(31)}-C_{R E F(1)}$ and a single-ended measured capacitance of $C_{M(1,1)}-C_{M(31,16)}$, the final output corresponding to $\mathrm{C}_{\mathrm{RCT}(1,1)}-\mathrm{C}_{\mathrm{RCT}(31,16)}$ can be obtained.

\subsection{Input Offset Cancellation and Capacitance Compensation Techniques}

Figure $7 \mathrm{a}-\mathrm{c}$ shows the detailed operation of the proposed $\mathrm{C} 2 \mathrm{~V}$ converter with the input offset cancellation technique. The $\mathrm{C} 2 \mathrm{~V}$ converter consists of positive and negative switched capacitor (SC) integrators and sampling switches at the inputs. When $\phi_{\mathrm{RST}}$ is high, the $V_{\mathrm{OP}}$ and $\mathrm{V}_{\mathrm{ON}}$ are reset to half of supply voltage. As $\phi_{\mathrm{RST}}$ falls, the charge on the mutual capacitance $C_{M}$ is transferred to the integral capacitors of $C_{\text {INTP }}$ and $C_{\text {INTN }}$. When $\phi_{1}$ is high and $V_{T X}$ is low, the charge proportional to $C_{M} V_{T X}$ is trapped on the ride-side of $C_{I N T P}$, increasing the $V_{O P}$. Then, when both $\phi_{2}$ and $V_{T X}$ are high, the negative charge of $C_{M} V_{T X}$ is transferred to the ride-side of $C_{I N T N}$, decreasing the $V_{O N}$. By repeating itself every TX sample, moving-average bandpass filtering in the analog domain is obtained [21]. 

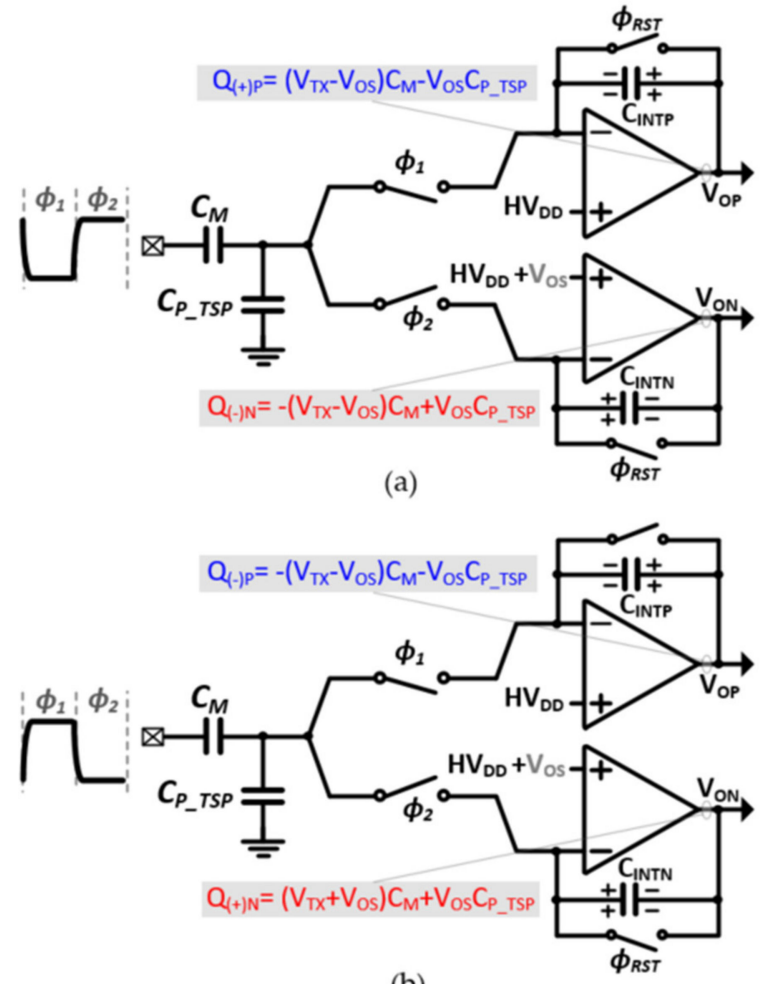

(b)

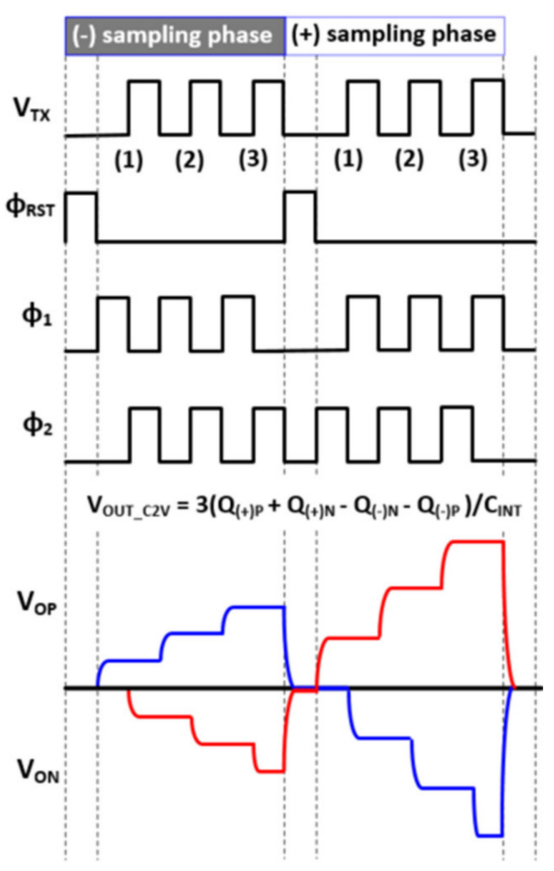

(c)

Figure 7. $\mathrm{C} 2 \mathrm{~V}$ configuration for (a) the negative offset sampling phase $\left(\phi_{1}=\right.$ high, $\mathrm{V}_{\mathrm{TX}}=$ low), (b) the positive offset sampling phase $\left(\phi_{1}=\right.$ high, $\mathrm{V}_{\mathrm{TX}}=$ high), and $(\mathbf{c})$ its timing diagram.

However, as the thickness of the AMOLED panel decreases, the TSP electrode becomes close to the display pixels, leading to the increased parasitic capacitance of $C_{P_{-} T S P}$. For encapsulation glass with a thickness of about $100 \mu \mathrm{m}$, the $\mathrm{C}_{\mathrm{P}_{-} \mathrm{TSP}}$ is increased to tens of picofarads. In this case, $\mathrm{C} 2 \mathrm{~V}$ can saturate as the number of moving average taps increases. Let us consider that the (+) input of a positive SC integrator in the $\mathrm{C} 2 \mathrm{~V}$ converter is higher than that of the negative SC integrator by $V_{O S}$. When $\phi_{1}$ is high, the $C_{P_{-} \text {TSP }}$ is charged to half of the supply voltage. Then, when $\phi_{1}$ is low and $\phi_{2}$ is high, the total charge on the $\mathrm{C}_{\mathrm{P}_{-} \mathrm{TSP}}$ increases by $\mathrm{C}_{\mathrm{P}_{-} \mathrm{TSP}} \mathrm{V}_{\mathrm{OS}}$, bringing an equal positive charge to the right-hand side of $\mathrm{C}_{\text {INTN }}$. Since these offsets can be varied from C2V[15:0], it degrades sensing accuracy and saturates the AFE. For example, with $100 \mu \mathrm{F}$ of $\mathrm{C}_{\mathrm{P}_{-} \mathrm{TSP}}, 2 \mu \mathrm{F}$ of $\mathrm{C}_{\mathrm{M}}, 3$, of TSP excitation signal, and $10, \mathrm{~V}$ of input offset voltage, an offset charge of $10 \mu \mathrm{C}$ is also introduced, which is one-sixth of the charge from mutual capacitance. To minimize a circuit's offset without any additional circuits, offset cancellation chopping is employed, which uses RX-correlated TSP driving.

The sensing phase is divided into two phases: the positive (+) offset sampling phase and the negative (-) offset sampling phase. During the negative sampling phase, when $\phi_{2}$ is high, $V_{T X}$ goes high. Therefore, a negative offset charge is stored on the $C_{\text {INTP }}$ while positive $\left(V_{T X}-V_{O S}\right) C_{M}$ is frozen on it, and vice versa for $C_{I N T N}$. The offset charge of $V_{O S} C_{M}$ is insignificant compared with that of $\mathrm{V}_{\mathrm{OS}} \mathrm{C}_{\mathrm{P}_{-} \mathrm{TSP}}$. During the positive sampling phase, when $\phi_{1}$ is high, $V_{T X}$ goes high. In this case, the negatives of both the $C_{M}$-charge and the offset charge are stored on the $C_{\text {INTP }}$, and vice versa for $C_{\text {INTN }}$ as well. To cancel the offset voltage at the $\mathrm{C} 2 \mathrm{~V}$ output, the $\mathrm{V}_{\mathrm{OP}}$ in the positive sampling phase adds to the $\mathrm{V}_{\mathrm{ON}}$ in the negative sampling phase. This can be enabled by reversing the connection in the 16-to-1 ADC Mux. The operational amplifier in the switched capacitor integrator is a two-stage class $\mathrm{AB}$ amplifier. Based on post-layout simulations, the amplifier achieves a DC gain of $61 \mathrm{~dB}$ and unity gain bandwidth of $12 \mathrm{MHz}$ while consuming $140 \mu \mathrm{W}$ power from a 3.3 supply. 
During the sensing phase for both the $\mathrm{T}_{\mathrm{VP}}$ and $\mathrm{T}_{\mathrm{HP}}$, the base capacitance, which is not unchanged by a finger touch and occupies most of the dynamic range of AFE, should be removed. The detailed operation of the compensation circuit used here is shown in Figure 8 [20]. When $\phi_{\mathrm{SC}}$ is high, the positive charge from the $\mathrm{I}_{\mathrm{SC}}$ is provided to the negative input of a switched capacitor integrator. When $\phi_{\mathrm{SK}}$ is high, the $\mathrm{I}_{\mathrm{SK}}$, which is equal to the $\mathrm{I}_{\mathrm{SC}}$, flows from its negative input. Since the TSP electrode is driven by AC signals, $\mathrm{I}_{\mathrm{SK}}$ and

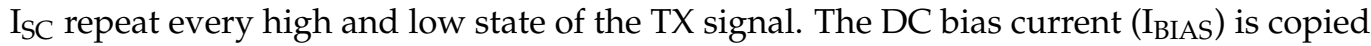
into the $\mathrm{T}_{\mathrm{P} 1}$ and $\mathrm{T}_{\mathrm{N} 1}$ and amplified $\mathrm{N}$ times by $\mathrm{T}_{\mathrm{P} 2}$ and $\mathrm{T}_{\mathrm{N} 1}$, respectively. In a practical design, a minimization of the mismatch between $\mathrm{I}_{\mathrm{SK}}$ and $\mathrm{I}_{\mathrm{SC}}$ is required. Thus, to minimize the channel length modulation effect, the $V_{D S}$ of $T_{P 1}$ and $T_{P 2}$, and the $V_{D S}$ of $T_{N 1}$ and $T_{N 2}$ are fixed to be equal using a regulated cascode feedback amplifier. Specifically, the phase of $\phi_{S K}$ or $\phi_{S C}$ leads that of $\phi_{1}$ or $\phi_{2}$, preventing a disturbance of the negative input voltage due to the charge transfer of $C_{M}$ when compensation starts. In a practical design, the delay is fixed to a time duration of $5 / \mathrm{f}_{\text {MAIN }}$ where $\mathrm{f}_{\text {MAIN }}$ is $40 \mathrm{MHz}$ or the operational clock of MCU in the designed IC.
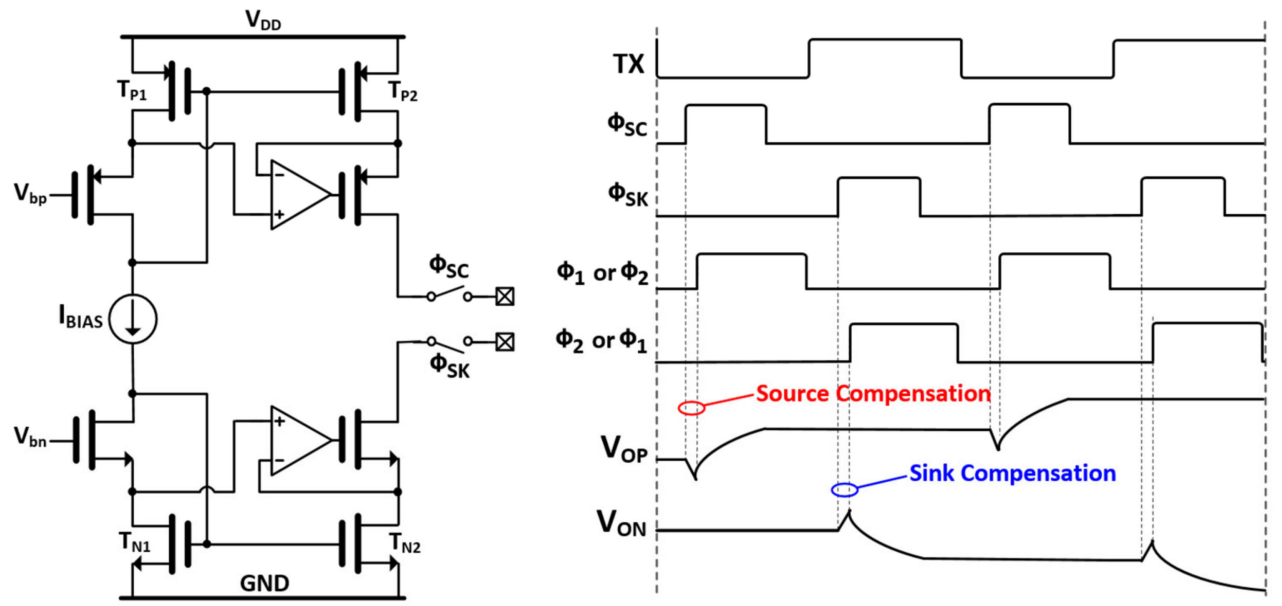

Figure 8. Circuit implementation of a capacitance compensator and its timing diagram.

\section{Result and Discussion}

The proposed readout IC was fabricated in a 130-nm CMOS process. The die photograph is shown in Figure 9. The active area, which includes the AFE, SAR ADC, bandgap reference $(B G R)$, and reference voltage generator $\left(V_{R E F}\right)$, is $3.4 \mathrm{~mm}^{2}$ and the full chip area is $8.02 \mathrm{~mm}^{2}$. TSP driving was synchronized with the display update, where its frame rate is measured to be $123 \mathrm{~Hz}$ with a $2400 \times 1080 \mathrm{FHD}$ plus 6.1-inch AMOLED panel. For a TSP having 31 TX and 16 RX electrodes, the total power consumption was $17.1 \mathrm{~W}$ with a 3.3, power supply. The power breakdown for the AFE was $6.4 \mathrm{~W}$. Operating the AFE only during part of a frame reduced its power consumption. Here, the AFE scan time was reduced to $3 \mathrm{~ms}$, including the reference capacitance and differential capacitance sensing phases.

Figure 10 shows the measured TX signal and C2V output in a TSP frame. The sensing phase for reference capacitance sensing was $320 \mu \mathrm{s}$, while the differential sensing time was set to be $2.45 \mathrm{~ms}$. Since touch coordinates should be extracted and provided to the application processor in a smartphone every TSP frame $(123 \mathrm{~Hz})$, digital signal processing time for interpolation, coordinate filtering, and baseline calibration is needed, and was set to be $4.3 \mathrm{~ms}$. Figure 11 provides the measured C2V output before the 16-to-1 ADC multiplexer when the input offset cancellation technique was applied. The TX frequency of $330 \mathrm{kHz}$ and a 16-repetition of the TX pulse were used for measurement. One orthogonal instant was divided into $(+)$ and $(-)$ sampling phases. The output voltage difference or offset voltage between these phases was $290 \mathrm{mV}$, which would be eliminated by adding outputs in each phase. To evaluate the effectiveness of sensing in $\mathrm{T}_{\mathrm{VP}}$, the reference capacitance 
$\left(\mathrm{C}_{\mathrm{ref}(1)} \sim \mathrm{C}_{\mathrm{ref}(31)}\right)$ was measured during both $\mathrm{T}_{\mathrm{VP}}$ and $\mathrm{T}_{\mathrm{HP}}$, as shown in Figure 12a where the $1-\mathrm{H}$ zebra image was updated. The SNR measured in the $\mathrm{T}_{\mathrm{VP}}$ was $22 \mathrm{~dB}$ higher than that of the $\mathrm{T}_{\mathrm{HP}}$, since any display signals related to the 1-H zebra image did not exist during $\mathrm{T}_{\mathrm{VP}}$. Figure $12 \mathrm{~b}, \mathrm{c}$ shows the differential and single-ended measured touch profile when $\mathrm{a}$ $4 \mathrm{~mm}$ diameter conductive rod was touched, with a display update from 1-H zebra image. Here, the touch data was RMS-averaged over 1000-frames. The noise values of the $\mathrm{T}_{\mathrm{HP}}$ $\left(\mathrm{N}_{\max }, \mathrm{N}_{\mathrm{avg}(31,16)}\right)$ were more than three times larger than that of the $\mathrm{T}_{\mathrm{VP}}$. The SNR for differential sensing was measured to $37 \mathrm{~dB}$, while that of single-ended sensing was $25.4 \mathrm{~dB}$. The SNR loss between the reference capacitance and the differential capacitance sensing was $9 \mathrm{~dB}$ (Figure 12a). This is because the coupled display noises at each RX electrode have a mismatch in phase and amplitude. The performance summary and comparison with previous works are shown in Table 1. Unlike other state-of-the-art works, it describes noise-immunity to display noise and suppresses it by $37 \mathrm{~dB}$ in the worst possible case. Although other works achieved better SNR, a metal-mesh TSP sensor has higher capacitive change according to touch. The metal-mesh sensor also has smaller TSP-series resistance, allowing the use of a higher TSP frequency.

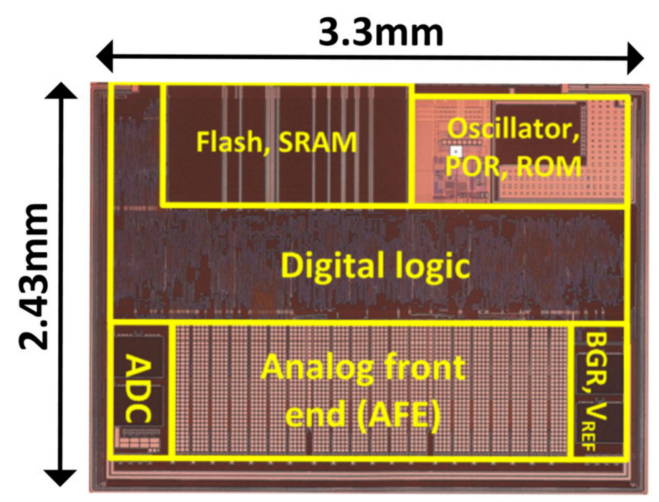

Figure 9. Die Photograph.

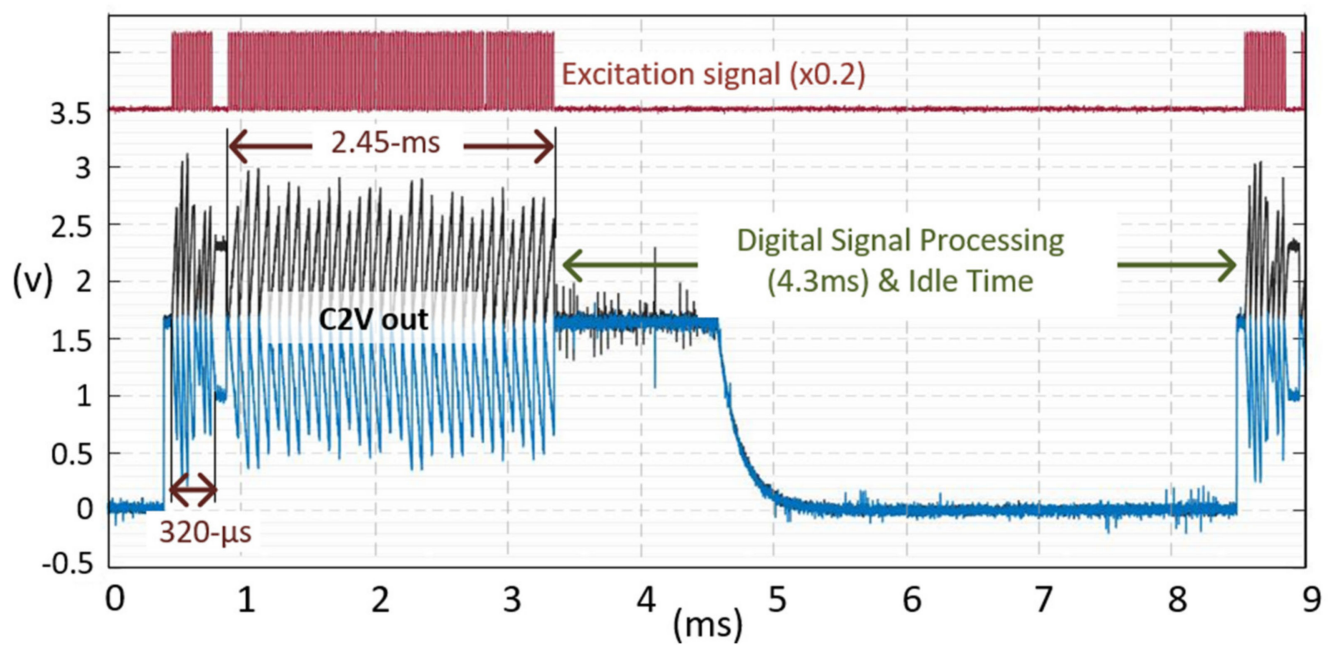

Figure 10. Measured waveforms of the TX signal (red) and the C2V output (black and blue) when synchronizing TSP and MDDI driving. 


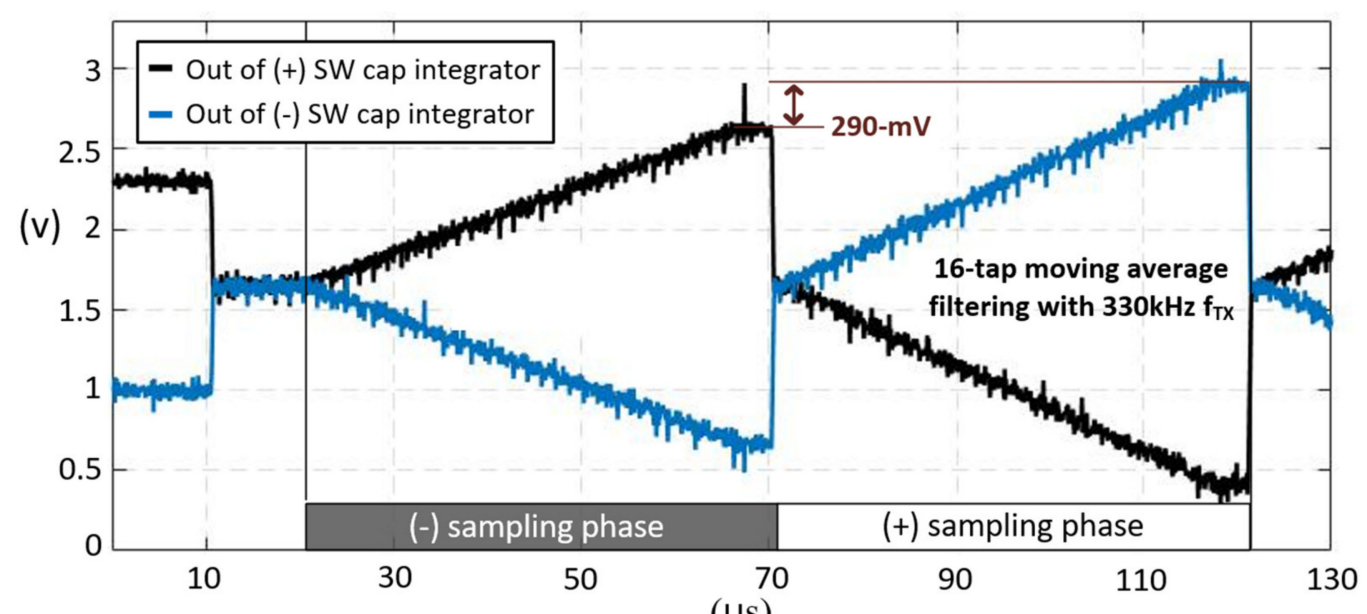

Figure 11. Measured waveforms of the C2V output when the chopping scheme is applied.

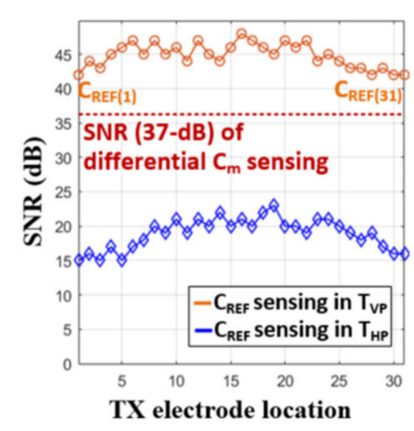

(a)

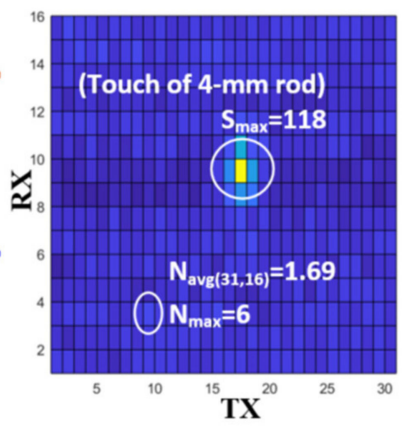

(b)

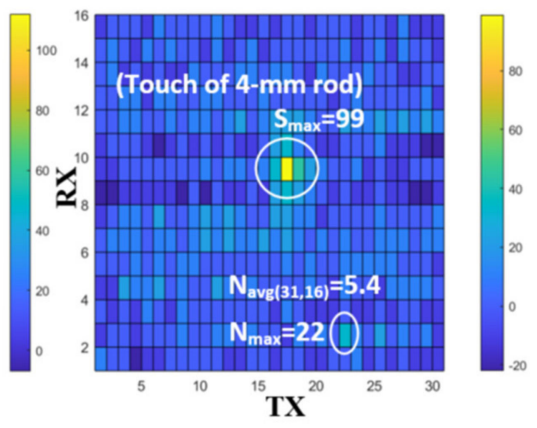

(c)

Figure 12. (a) Comparison of SNR for reference capacitance $\left(C_{R E F}\right)$ during $T_{V P}$ and $T_{H P}$, and the measured RMS touch profile of (b) the proposed differential TSP sensing and (c) conventional single-ended sensing.

Table 1. Performance summary and comparison to previous works.

\begin{tabular}{|c|c|c|c|c|}
\hline & This Work & Ref. [14] & Ref. [15] & Ref. [22] \\
\hline Process & $130 \mathrm{~nm}$ & $180 \mathrm{~nm}$ & $180 \mathrm{~nm}$ & $350 \mathrm{~nm}$ \\
\hline Electrodes & $31 \mathrm{TX}$ and $16 \mathrm{RX}$ & $16 \mathrm{TX}$ and $33 \mathrm{RX}$ & $36 \mathrm{TX}$ and $64 \mathrm{RX}$ & $15 \mathrm{TX}$ and $10 \mathrm{RX}$ \\
\hline Scan rate & $\begin{array}{l}330 \mathrm{~Hz} \\
\text { (Synchronous to } 120 \mathrm{~Hz} \text { AMOLED) }\end{array}$ & $120 \mathrm{~Hz}$ & $85 \sim 385 \mathrm{~Hz}$ & $250 \mathrm{~Hz}$ \\
\hline Power Consumption & $\begin{array}{c}6.4 \mathrm{~mW} \text { (AFE) } \\
17.1 \mathrm{~mW} \text { (Full chip) }\end{array}$ & $17.8 \mathrm{~mW}$ & $67.7 \mathrm{~mW}$ & $12.8 \mathrm{~mW}$ \\
\hline Chip area & $\begin{array}{c}3.4 \mathrm{~mm}^{2} \text { (AFE) } \\
8.02 \mathrm{~mm}^{2} \text { (Full chip) }\end{array}$ & $7.1 \mathrm{~mm}^{2}$ & $36 \mathrm{~mm}^{2}$ & $4.89 \mathrm{~mm}^{2}$ \\
\hline SNR & $\begin{array}{l}46 \mathrm{~dB} \text { without display noise } \\
37 \mathrm{~dB} \text { with zebra pattern in } 120 \mathrm{~Hz} \text { AMOLED }\end{array}$ & $57 \mathrm{~dB}$ & $54 \mathrm{~dB}$ & $43 \mathrm{~dB}$ \\
\hline TSP Type & ITO & Metal Mesh & ITO & Metal Mesh \\
\hline
\end{tabular}

\section{Conclusions}

This article describes a display-noise-immune TSP readout system with synchronization of touch and display driving. It reduces the display noise by more than 4 times and has a compact structure without any additional circuits for differential sensing. The tradeoff between dynamic range and crosstalk is eased by introducing different orthogonal encoding for reference and differential sensing. 
This technique is applicable to other display architectures. It can be easily adopted in an LCD or flexible AMOLED where the vertical porch and horizontal update instants exist.

Funding: The present research was conducted with funding by the Research Grant of Kwangwoon University in 2021.

Conflicts of Interest: The author declares no conflict of interest.

\section{References}

1. Galaxy S21 Ultra 5G. Available online: https://www.samsung.com/global/galaxy/galaxy-s21-ultra-5g/specs/ (accessed on 19 June 2021).

2. Galaxy Tab S7. Available online: https:/ / www.samsung.com/global/galaxy/galaxy-tab-s7/ (accessed on 19 June 2021).

3. How Do You Use Always on Display Mode on Galaxy S20, S20+, S20 Ultra, and Z Flip? Available online: https:/ /www.samsung. com/global/galaxy/what-is/always-on-display/ (accessed on 19 June 2021).

4. David, W. Capacitive Sensing: Ins and Outs of Active Shielding. Available online: https://www.ti.com/lit/an/snoa926a/snoa9 26a.pdf?ts=1627473795125\&ref_url=https\%253A\%252F\%252Fwww.google.com\%252F (accessed on 25 July 2021).

5. Kim, H.R.; Choi, Y.-K.; Byun, S.-H.; Kim, S.-W.; Choi, K.-H.; Ahn, H.-Y.; Park, J.-K.; Lee, D.-Y.; Cho, H.-H.; Yu, J.-S.; et al. A Mobile-Display-Driver IC Embedding a Capacitive-Touch-Screen Controller System. In Proceedings of the International Solid-State Conference Digest of Technical Papers (ISSCC), San Francisco, CA, USA, 7-11 February 2010; pp. 114-116.

6. Hotelling, S.P.; Krah, C.H.; Huppi, B.Q. Multipoint Touch Surface Controller. U.S. Patent 8279 180, 2 October 2012.

7. Huang, S.-L.; Hung, S.-Y.; Chen, C.-P. Frequency hopping and parallel driving with random delay especially suitable for the charger noise problem in mutual-capacitive touch applications. IEEE Access 2018, 7, 3980-3993. [CrossRef]

8. Park, J.-E.; Lim, D.-H.; Jeong, D.-K. A reconfigurable 40-to-67 dB SNR, 50-to-6400 Hz frame-rate, column-parallel readout IC for capacitive touch-screen panels. IEEE J. Solid-State Circuits 2014, 49, 2305-2318. [CrossRef]

9. Hamaguchi, M.; Nago, A.; Miyamoto, M. A 240 Hz-Reporting Rate $143 \times 81$ Mutual-Capacitance Touch-Sensing Analog Front-End IC with $37 \mathrm{~dB}$ SNR for $1 \mathrm{~mm}$-diameter Stylus. In Proceedings of the International Solid-State Circuits Conference Digest of Technical Papers (ISSCC), San Francisco, CA, USA, 9-13 February 2014; pp. 214-216.

10. Kim, K.-D.; Kang, S.; Choi, Y.-K.; Lee, K.-H.; Lee, J.-C.; Choi, M.; Ko, K.; Jung, J.; Park, N.; Park, H.; et al. A Fully-Differential Capacitive Touch Controller with Input Common-Mode Feedback for Symmetric Display Noise Cancellation. In Proceedings of the IEEE Symposium on VLSI Circuits, Honolulu, HI, USA, 10-13 June 2014; pp. 1-2.

11. Lee, C.-J.; Park, J.K.; Seo, H.-E.; Huh, J.; Chun, J.-H. Fully differential touch screen controller with wide input dynamic range for thin displays. Sensors 2020, 20, 837. [CrossRef] [PubMed]

12. Klein, K. Noise Immunity of Touchscreen Devices. Available online: http://www.cypress.com/file/120641/download (accessed on 25 July 2021).

13. Nam, H.; Seol, K.-H.; Lee, J.; Cho, H.; Jung, S.W. Review of capacitive touchscreen technologies: Overview, research trends, and machine learning approaches. Sensors 2021, 21, 4776. [CrossRef] [PubMed]

14. Lee, C.-J.; Park, J.; Seo, H.-E.; Huh, J.; Chun, J.-H. A mutual capacitance touch readout IC with $64 \%$ reduced-power adiabatic driving over heavily coupled touch ccreen. IEEE J. Solid-State Circuits 2019, 54, 1694-1704.

15. Park, J.-E.; Park, J.; Hwang, Y.-H.; Oh, J.; Jeong, D.-K. A noise-immunity-enhanced analog front-end for $36 \times 64$ touch-screen controllers with 20-VPP noise tolerance at $100 \mathrm{kHz}$. IEEE J. Solid State Circuits 2019, 54, 1497-1510. [CrossRef]

16. Ko, S. A-24 dB in-band noise-immunity mutual capacitance readout system for variable refresh rate of active-matrix OLED display. IEEE Trans. Circuits Syst. I Regul. Pap. 2020, 67, 1126-1135. [CrossRef]

17. Dinan, E.H.; Jabbari, B. Spreading codes for direct sequence CDMA and wideband CDMA cellular networks. IEEE Commun. Mag. 1998, 36, 48-54. [CrossRef]

18. Shin, H.; Ko, S.; Jang, H.; Yun, I.; Lee, K. A 55 dB SNR with 240 Hz Frame Scan Rate Mutual Capacitor $30 \times 24$ Touch-Screen Panel Read-Out IC Using Code-Division Multiple Sensing Technique. In Proceedings of the International Solid-State Circuits Conference Digest of Technical Papers (ISSCC), San Francisco, CA, USA, 17-21 February 2013; pp. 388-390.

19. Golomb, S.W.; Scholtz, R.A. Generalized barker sequences. IEEE Trans. Inf. Theory 1965, 11, 533-537. [CrossRef]

20. Ko, S. An Automatic Ear Detection Technique in Capacitive Sensing Readout IC Using Cascaded Classifiers and Hovering Function. In Proceedings of the Symposium on VLSI Circuits, Kyoto, Japan, 9-14 June 2019.

21. Ko, S.; Shin, H.; Lee, J.; Jang, H.; So, B.-C.; Yun, I.; Lee, K. Low Noise Capacitive Sensor for Multi-Touch Mobile Handset's Applications. In Proceedings of the Asian Solid-State Circuits Conference, Beijing, China, 8-10 November 2010; pp. 1-4.

22. Lee, C.-J.; Park, J.K.; Piao, C.; Seo, H.-E.; Choi, J.; Chun, J.-H. Mutual capacitive touch screen controller for ultrathin display with extended signal passband using negative capacitance. Sensors 2018, 18, 3637. [CrossRef] [PubMed] 